\title{
Criminologie
}

Processus de changement des adolescents auteurs de violences sexuelles Évaluation, indicateurs cliniques et perspectives de soin dans le cadre de thérapies de groupe

Processes of change for teenage sex offenders

Evaluation, clinical indicators, and perspectives on care in the context of group therapy

El proceso de cambio de los adolescentes autores de violencias sexuales Evaluación, indicadores clínicos y perspectivas de cuidado en el marco de terapias de grupo

Pascal Roman, Thérèse Cuttelod, Sylvie Espeil-Marc, Dorothée Gorry, Frédérique Lavèze et Magali Ravit

Volume 52, numéro 2, automne 2019

URI : https://id.erudit.org/iderudit/1065864ar

DOI : https://doi.org/10.7202/1065864ar

Aller au sommaire du numéro

Éditeur(s)

Les Presses de l’Université de Montréal

ISSN

0316-0041 (imprimé)

1492-1367 (numérique)

Découvrir la revue

Citer cet article

Roman, P., Cuttelod, T., Espeil-Marc, S., Gorry, D., Lavèze, F. \& Ravit, M. (2019).

Processus de changement des adolescents auteurs de violences sexuelles :

évaluation, indicateurs cliniques et perspectives de soin dans le cadre de

thérapies de groupe. Criminologie, 52(2), 267-299.

https://doi.org/10.7202/1065864ar
Résumé de l'article

Cet article présente les principales avancées issues d'une recherche menée conjointement sur deux sites cliniques, en Suisse et en France, et consacrée à l'évaluation des processus de changement des adolescents auteurs de violences sexuelles. L'objectif est de dégager les principaux indicateurs cliniques de ces changements, à partir d'une étude longitudinale portant sur un petit nombre d'adolescents $(N=9)$, engagés dans une démarche psychothérapeutique sous mandat judiciaire en groupe. L'étude s'appuie sur une démarche qualitative, qui associe principalement deux types de recueil de données cliniques : épreuves projectives en test-retest d'une part et enregistrement des verbatims de séances de groupe d'autre part. À partir de l'analyse croisée de ces données, un certain nombre d'indicateurs cliniques peuvent être mis en évidence, qui permettent d'esquisser une modélisation des processus de changement chez les adolescents. Au-delà, les éléments de compréhension qui émergent de la recherche permettent de tracer des perspectives pour le soin psychothérapeutique des adolescents. 


\section{Processus de changement des adolescents auteurs de violences sexuelles}

\section{Évaluation, indicateurs cliniques et perspectives de soin dans le cadre de thérapies de groupe}

\author{
Pascal Roman ${ }^{1}$ \\ Professeur de psychologie clinique, \\ psychopathologie et psychanalyse \\ Université de Lausanne \\ pascal.roman@unil.ch \\ Thérèse Cuttelod \\ Psychothérapeute reconnue au niveau fédéral \\ Association ESPAS \\ therese.cuttelod@espas.info \\ Sylvie Espeil-Marc \\ Infirmière diplômée d'État \\ Centre Hospitalier Alpes Isère \\ sespeilmarc@ch-alpes-isere.fr
}

\author{
Dorothée Gorry \\ Psychologue clinicienne \\ Centre Hospitalier Le Vinatier \\ dorothee.gorry@ch-le-vinatier.fr \\ Frédérique Lavèze \\ Psychologue clinicienne \\ CRIAVS-RA, Centre Ressource Claude Balier \\ Consultation Alliance Adolescents \\ Centre Hospitalier Alpes Isère \\ f.laveze@criavs-ra.org \\ Magali Ravit \\ Professeure de psychopathologie \\ et psychologie clinique \\ Institut de psychologie - CRPPC \\ Université Lumière-Lyon 2 \\ magali.ravit@wanadoo.fr
}

RÉSUMÉ - Cet article présente les principales avancées issues d'une recherche menée conjointement sur deux sites cliniques, en Suisse et en France, et consacrée à l'évaluation des processus de changement des adolescents auteurs de violences sexuelles. L'objectif est de dégager les principaux indicateurs cliniques de ces changements, à partir d'une étude longitudinale portant sur un petit nombre d'adolescents $(\mathrm{N}=9)$, engagés dans une démarche psychothérapeutique sous mandat judiciaire en groupe. L'étude s'appuie sur une démarche qualitative, qui associe principalement deux types de recueil de données cliniques: épreuves projectives en test-retest d'une part et enregistrement des verbatims de séances de groupe d'autre part. À partir de l'analyse croisée de ces données, un certain nombre d'indicateurs cliniques peuvent être mis en

1. Institut de Psychologie - LARPsyDIS, Université de Lausanne, Bâtiment Geopolis, 1015, Lausanne, Suisse. 
évidence, qui permettent d'esquisser une modélisation des processus de changement chez les adolescents. Au-delà, les éléments de compréhension qui émergent de la recherche permettent de tracer des perspectives pour le soin psychothérapeutique des adolescents.

MOTS CLÉS - Adolescent, violences sexuelles, psychothérapie, indicateurs cliniques, évaluation.

\section{Introduction}

La question des violences sexuelles à l'adolescence agite les milieux professionnels dans le champ social, judiciaire et clinique. L'incompréhension devant des agirs dont la violence inquiète se double d'une difficulté à appréhender le traitement psychothérapeutique de ces adolescents. Pour différentes raisons, qui tiennent à la fois aux spécificités cliniques et aux échos des agirs violents sexuels à l'adolescence chez les professionnels (Roman, 2012, 2016), le choix des professionnels du soin psychique s'oriente le plus souvent vers des dispositifs de groupe: ceux-ci sont reconnus pertinents puisqu'ils permettent de soutenir une «diffraction du transfert» (Kaës, 1993) ainsi que le dégagement des effets de séduction ou de persécution à l'œuvre en relation duelle. Le groupe permet également de valoriser un appui sur les pairs, précieux dans le contexte de fragilité narcissique de ces adolescents, ainsi que d'autoriser expression et circulation de la parole et des représentations.

C'est dans ce contexte que s'inscrit notre souhait de mieux comprendre les processus de changement des adolescents mis en cause et condamnés pour des transgressions sexuelles. Cette démarche prend place dans le cadre plus large des recherches sur l'évaluation des pratiques et des dispositifs psychothérapeutiques (Brun, Roussillon et Attigui, 2016; Fischman, 2009) et vise à fournir aux professionnels des repères pour le traitement des adolescents engagés dans des agirs violents sexuels.

En effet, au-delà des études qui permettent d'affiner l'approche de la problématique des adolescents auteurs de violences sexuelles du point de vue psychocriminologique et du point de vue psychodynamique (de Becker, 2009; Gamache, Diguer, Laverdière et Rousseau, 2014; Lemitre et Coutanceau, 2006; Pelladeau et Marchand, 2016; Pelladeau, Roques et Pommier, 2015; Roman, 2012), il apparaît nécessaire de procéder à l'évaluation des processus psychiques mobilisés chez les adolescents engagés dans un traitement psychothérapeutique. Cette 
évaluation vise à comprendre les ressorts psychodynamiques en jeu au décours du processus psychothérapeutique et à apprécier la contribution $\mathrm{du}$ dispositif de soin aux processus de changement.

La spécificité de cette recherche est triple $\left.{ }^{2}: 1\right)$ elle se déploie sur deux terrains cliniques, en Suisse et en France; 2) elle mobilise des données cliniques bétérogènes, issues de la pratique de l'examen psychologique (épreuves projectives) en test-retest et de l'enregistrement de séances de groupe; 3) elle se focalise sur l'analyse des processus psychiques engagés dans le traitement psychothérapeutique au travers d'une méthodologie de type longitudinal, caractérisée par une durée relativement réduite de la prise en compte de l'évolution.

\section{Revue de littérature}

Les publications sur l'évaluation des processus de changement dans le cadre de psychothérapies d'adolescents auteurs de violences sexuelles sont relativement limitées, en lien avec une récence de cette problématique sur la scène sociale. Peu d'études ont évalué la thérapie de groupe en elle-même, et la majorité des articles présente des entretiens de groupe en parallèle à un traitement individuel et à une thérapie familiale. McGarvey et Lenaghan (1996) laissent supposer, en effet, que la thérapie de groupe avec des auteurs de violences sexuelles mineurs est efficace à condition d'être administrée conjointement avec une thérapie individuelle. Dans la littérature francophone, la thérapie de groupe est également souvent combinée à un suivi individuel (Auguste et al., 2010; Collart, Depauw et Heremans, 2002; Ellerby, McGrath, Cumming, Burchard et Zeoli, 2010; Emeraud, 2001; Laforest et Paradis, 1990; Lemitre et Coutanceau, 2006). Selon Perkins, Hammond, Coles et Bishopp (1998), l'approche psychodynamique, centrée sur les fantasmes, pourrait être utile dans le traitement des auteurs de violences sexuelles en combinaison avec d'autres formes de psychothérapie. Aujourd'hui, de nombreux auteurs semblent penser qu'une prise en charge multidimensionnelle, incluant des dispositifs individuels, de

2. Recherche EvAdoGroupe (Évaluation des processus de changement des adolescents auteurs de violences sexuelles pris en charge dans des groupes thérapeutiques), réalisée dans le cadre du LARPsyDIS, Institut de psychologie, Université de Lausanne, et financée par le Service de protection de la jeunesse du canton de Vaud (Lausanne, Suisse) et par le CRIAVS Rhône-Alpes (Grenoble, France). Ce protocole de recherche a été approuvé par la Commission d'éthique de la recherche du canton de Vaud (Suisse) le 24 octobre 2013. 
groupe, voire une thérapie familiale, conduirait aux meilleurs résultats (Grant et al., 2009; McGarvey et Lenaghan, 1996).

Il est proposé que la thérapie de groupe avec les adolescents auteurs de violences sexuelles permette un apprentissage vicariant en appui sur les autres participants (Ertl et McNamara, 1997; Grant et al., 2009; Lemitre et Coutanceau, 2006). Une autre caractéristique de ce dispositif est qu'il permet à la fois le soutien et la confrontation par les pairs (Levenson, Macgowan, Morin et Cotter, 2009; McGarvey et Lenaghan, 1996; Viens, Tourigny, Lagueux et Loméga, 2011). La thérapie groupale pourrait favoriser le changement grâce aux interactions et dynamiques entre participants, puisqu'il semblerait qu'il soit plus acceptable pour l'auteur d'affronter ses pairs que le thérapeute. De plus, le soutien par les pairs dans la thérapie de groupe permet de créer un environnement sécurisant qui encourage la révélation de soi et réduit les secrets (Auguste et al., 2010; Dwyer et Boyd, 2009; Perkins et al., 1998). Cet environnement protégerait également le thérapeute de l'emprise des représentations de l'auteur du délit (Perkins et al., 1998).

Swart, Bass et Apsche (2015) notent la difficulté, dans la prise en charge des adolescents auteurs de violences sexuelles, de mettre en avant la dimension transgressive des actes, surtout lorsqu'ils sont commis «sans violence» ou à titre «expérimental» (Swart et al., 2015). Les auteurs insistent sur le fait que le traitement psychothérapeutique représente une voie prometteuse pour la prise en charge de ces adolescents, tout en relevant l'éclectisme des propositions thérapeutiques. Par ailleurs, ils relèvent que peu de dispositifs thérapeutiques font l'objet d'une évaluation du point de vue de leur efficacité. Lorsque la parole est donnée aux adolescents auteurs de violences sexuelles au sujet des programmes en groupe, ces derniers remarquent un changement dans le contrôle de soi (impulsivité) et en ce qui concerne leurs émotions, particulièrement en ce qui concerne le sentiment de colère (Derezotes, 2000 ; Grant et al., 2009). L'autoévaluation des adolescents ayant bénéficié d'un programme de traitement multisystémique, incluant des entretiens individuels, de famille et de groupe, rapporte des changements dans le contrôle de soi, la prise de responsabilité de leurs actes et le sentiment de colère à l'égard d'autrui (Grant et al., 2009). Le traitement n'a cependant pas permis d'améliorer les scores des jeunes participants au niveau de l'empathie pour les victimes. Dans les résultats de Grant et al. (2009), les adolescents attribuent spécifiquement au groupe l'effet positif de la diminution de leur sentiment d'isolement. 
Par ailleurs, des travaux récents (Smaniotto, Réveillaud et Félicier, 2014) centrés sur une prise en charge en groupe basée sur une médiation confirment l'importance de celui-ci pour la mobilisation des ressources des adolescents auteurs de violences sexuelles: espace sécurisant dans lequel s'expérimente la relation à l'autre, le groupe thérapeutique favorise le développement de l'empathie.

Enfin, Viens et al. (2011) ont mené une étude qui compare un groupe contrôle (adolescents auteurs de violences sexuelles au bénéfice d'un traitement usuel dans un centre jeunesse du Québec) avec un groupe expérimental (adolescents participant à un groupe de thérapie spécialisée parallèlement au traitement usuel). De nombreuses variables ont été mesurées (gestion de situations stressantes, habiletés sociales, compréhension du processus d'agression, anxiété, dépression, stress post-traumatique, préoccupations sexuelles, dissociation, sentiments de solitude et de colère). Le groupe expérimental a obtenu des résultats significativement plus élevés que le groupe contrôle sur quatre variables seulement: compréhension du processus d'agression, reconnaissance du caractère prémédité des actes, reconnaissance d'un intérêt sexuel inapproprié et attitudes sexuelles. Aucune différence n'est relevée entre les deux groupes pour les autres variables, laissant supposer que tous deux ont évolué de manière similaire dans le temps.

À la lumière des connaissances actuelles sur l'intérêt et l'efficacité des psychothérapies de groupe pour adolescents auteurs de violences sexuelles, peu de données sont disponibles concernant spécifiquement les processus qui sous-tendent le changement; ceux-ci méritent d'être appréhendés dans le double registre de l'intrapsychique et de l'intersubjectif.

Sur un plan méthodologique, on peut identifier deux grands courants de la recherche sur l'évaluation des psychothérapies, qui suivent la partition des paradigmes de recherche décrits par Perron (2010): 1) les recherches s'inscrivant dans la voie de la recherche expérimentale, selon le paradigme de la médecine fondée sur les faits (evidence-based medicine), à partir d'études randomisées (Fischman, 2009), portant sur des cohortes de patients et visant à démontrer l'efficacité des dispositifs psychothérapeutiques; et 2) les recherches empruntant la voie de la démarche clinique, selon le paradigme constructiviste et compréhensif (dans lequel s'inscrit la psychanalyse), où l'accent est mis sur l'analyse de cas singuliers afin de montrer l'efficacité des dispositifs psychothérapeutiques, cas dont la mise en perspective permet d'esquisser une généralisation (Brun et al., 2016). 


\section{Méthodologie}

Cette étude s'inscrit clairement dans le second courant de la recherche sur l'évaluation des psychothérapies, centrée sur les processus (Brun et Roussillon, 2016), qui met l'accent sur le déploiement du traitement et son évolution, davantage que sur l'efficacité à proprement parler de la psychothérapie telle qu'habituellement considérée en termes de réitération ou non d'agirs transgressifs.

Il s'agit d'une recherche multicentrique : la population de recherche est constituée à partir de deux groupes thérapeutiques dont les animateurs sont impliqués dans l'équipe de recherche ${ }^{3}$. Chacun des groupes possède sa particularité, liée à la culture et aux pratiques des équipes: un groupe de parole, qui se déploie à partir de la proposition de thématiques en lien avec les agirs sexuels transgressifs, et un groupe de psychodrame psychanalytique. La présentation de la recherche aux adolescents est réalisée par les professionnels cliniciens, qui assurent conjointement avec le chercheur le recueil du consentement (adolescent) et des autorisations (parents) par le psychologue chercheur. L'implication des équipes dans la recherche constitue un point central dans l'implantation de la recherche et la qualité du recueil des données.

Les critères d'inclusion pour le recrutement des adolescents sont les suivants: la mise en cause ou condamnation pour des actes d'ordre sexuel au temps de la minorité (avant 18 ans) et être âgé de 12 à 21 ans au moment de l'inclusion dans la recherche. Il n'y a pas de critères d'exclusion spécifiques, liés aux compétences cognitives de l'adolescent ou à l'existence d'un éventuel diagnostic psychopathologique. Par ailleurs, les adolescents sont libres de quitter la recherche à tout moment.

$\mathrm{Au}$ regard de l'orientation méthodologique de la recherche, les outils cliniques mobilisés s'inscrivent dans le courant des approches compréhensives.

Sur le plan de l'évaluation du fonctionnement psychique:

- les épreuves projectives de Rorschach et du Thematic Apperception Test (TAT), considérées dans une approche et une méthodologie s'inscrivant dans une orientation psychanalytique et dont les qualités cliniques et herméneutiques ne sont plus à démontrer (Emmanuelli 
et Azoulay, 2008; Roman, 2015). Ces épreuves appartiennent aux épreuves projectives verbales, et reposent sur une invitation à une production relevant de l'imaginaire (réponse pour le test de Rorschach, récit pour le TAT), en appui sur un matériel standardisé ambigu, non figuratif (Rorschach) ou figuratif (TAT).

- le Questionnaire d'investigation clinique pour les adolescents auteurs de violences sexuelles (QICAAICS), dans sa version française ou suisse, inspiré des travaux de Balier, Ciavaldini et Girard-Khayat (1996), permet la rencontre de l'adolescent dans le contexte d'un entretien structuré (Roman et Ravit, 2010), en appui sur une sollicitation systématique centrée sur un certain nombre de thématiques ${ }^{4}$; les données recueillies permettent de contextualiser tout particulièrement les aspects liés aux agirs violents sexuels et à la dynamique familiale, mais permettent également de repérer les éventuels changements intervenant entre les deux temps de rencontre.

Sur le plan de l'évaluation des implications au sein du groupe, le choix de l'enregistrement audiophonique de séances de groupe, secondairement transcrit, constitue le matériel clinique sur lequel repose l'analyse des mouvements qui accompagnent le déroulement du soin.

Le plan de recherche prévoit différents temps dans le recueil des données:

1. La passation des tests de Rorschach et de TAT inaugure l'entrée dans la recherche et elle fait suite à la proposition d'un questionnaire d'investigation clinique, QICAAICS ou QICAAICS-CH (Roman et Ravit, 2010) selon le pays où se déroule la passation ${ }^{5}$. La passation des épreuves projectives est répétée environ neuf mois plus tard.

2. L'enregistrement audio des séances de groupe, réalisé à intervalles de trois mois, dont les adolescents sont informés préalablement.

Pour être inclus dans la population de recherche, les adolescents doivent avoir réalisé le test-retest (T-RT) des deux épreuves projectives et avoir été présents à trois temps d'enregistrement des séances de groupe

4. Le QICAAICS investigue successivement la rencontre avec la justice, les faits reprochés, la description de l'acte, la perception de l'acte par l'adolescent, la dynamique familiale, les relations amicales, la vie affective et sexuelle, la vie relationnelle, la dimension somatique.

5. Les éléments cliniques issus du questionnaire d'investigation clinique ne seront pas présentés de manière systématique dans cet article; ils possèdent essentiellement une fonction de médiation dans la rencontre avec les adolescents et de contextualisation pour l'analyse des données. 
minimum. Sur les treize adolescents présents dans les deux groupes dans la période de la recherche, seuls neuf répondent finalement à ces critères.

\section{Épreuves projectives}

Les protocoles de Rorschach et de TAT (T pour treize adolescents; T-RT pour neuf adolescents) ont fait l'objet d'une transcription, puis d'une cotation en double aveugle. Les protocoles feront l'objet d'une analyse sur la base des observatoires considérés comme significatifs pour l'évaluation des potentiels de changement des adolescents auteurs de violences sexuelles pris en charge dans des groupes thérapeutiques, en appui sur les données issues de la littérature (Roman et Melchiorre, 2015).

La pertinence de la démarche de test-retest dans le champ de la recherche clinique est attestée par un certain nombre de publications et considérée comme une modalité d'évaluation des processus de changement. Avec Piotrowski (1936) déjà, l'approche de l'évolution du fonctionnement psychique, en appui sur l'épreuve de Rorschach, s'inscrit dans une telle démarche. Dans les dernières décennies, tant en France qu'aux États-Unis, l'évaluation des psychothérapies mobilise un tel dispositif (Andronikof et Réveillère, 2004; Weiner et Exner, 1991), ces travaux se situant quasi exclusivement dans la perspective du système intégré. La recherche dirigée par Chabert et al. (1990) sur l'évolution de jeunes patients souffrant de troubles psychotiques constitue par ailleurs un modèle particulièrement pertinent pour soutenir notre propre démarche d'analyse.

L’analyse des données issues des épreuves projectives est réalisée dans une démarche de type hypothético-déductif. Elle s'appuie sur l'identification de cinq observatoires cliniques construits en appui sur l'étude de la littérature scientifique consacrée à la dynamique psychique des adolescents auteurs de violences sexuelles. Chacun de ces observatoires est caractérisé par un certain nombre d'indicateurs cliniques (voir Tableau 1), respectivement identifiés pour le Rorschach et le TAT: 1) Approche de la dynamique entre réalité interne et réalité externe; 2) Expression et traitement des angoisses primaires de différenciationséparation; 3) Mise à l'épreuve du registre odipien de conflictualité, en lien avec le registre de confortation narcissique; 4) Qualité de la liaison affect - représentation et dégagement de l'envahissement perceptif ou traumatique; et 5) Prise en compte des indices de la symbolisation de l'acte 
(de l'agir comme risque pour le processus de symbolisation à l'agir comme soutien).

Les épreuves de Rorschach et du TAT ont été proposées en test (T) - retest (RT) à huit mois d'intervalle en moyenne. L'axe de lecture du matériel clinique est donc comparatif et vise à faire émerger, pour chacun des observatoires, les grandes lignes d'évolution entre les deux temps de la passation.

TABLEAU 1

Synthèse des indicateurs des processus de changement aux épreuves projectives

\begin{tabular}{|l|l|l|}
\hline Observatoire & \multicolumn{1}{|c|}{ Rorschach } & \multicolumn{1}{c|}{ TAT } \\
\hline $\begin{array}{l}\text { 1. Dynamique réalité } \\
\text { interne/réalité externe }\end{array}$ & $\begin{array}{l}\text { G, F+\%, K, Ban et } \\
\text { refus }\end{array}$ & $\begin{array}{l}\text { Répartition procédés } \\
\text { élaboration des récits }\end{array}$ \\
\hline $\begin{array}{l}\text { 2. Angoisses séparation/ } \\
\text { différenciation }\end{array}$ & $\begin{array}{l}\text { Traitement du blanc et } \\
\text { de la symétrie }\end{array}$ & $\begin{array}{l}\text { Traitement sollicitation } \\
\text { dépressive (planche 3BM) }\end{array}$ \\
\hline $\begin{array}{l}\text { 3. Tension registre } \\
\text { œdipien versus } \\
\text { narcissique }\end{array}$ & $\begin{array}{l}\text { Qualité investissement } \\
\text { réponses humaines }\end{array}$ & $\begin{array}{l}\text { Élaboration triangulation } \\
\text { œdipienne (planches 2, 6 } \\
\text { et 7BM) }\end{array}$ \\
\hline $\begin{array}{l}\text { 4. Liison affect/ } \\
\text { représentation }\end{array}$ & Refus, réponses FC & $\begin{array}{l}\text { Souplesse des récits et des } \\
\text { procédés d'élaboration }\end{array}$ \\
\hline $\begin{array}{l}\text { 5. Symbolisation } \\
\text { de l'acte }\end{array}$ & $\begin{array}{l}\text { Destin des } \\
\text { kinesthésies }\end{array}$ & $\begin{array}{l}\text { Investissement de l'acte: } \\
\text { de l'agir à l'action. Focus } \\
\text { sur planches 8BM, 13MF, 16 }\end{array}$ \\
\hline
\end{tabular}

Séances de groupe

Les deux groupes sur lesquels porte la recherche proposent des modalités de rencontre différentes avec les adolescents: groupe de parole, pour une part thématisé pour l'un, groupe de psychodrame pour l'autre. Il s'agit de groupes coanimés (deux à trois thérapeutes), ouvert pour le premier, fermé pour le second, comportant entre trois et six adolescents. Le critère d'inclusion est celui de la condamnation par la justice des mineurs pour des infractions à caractère sexuel; parmi les critères d'exclusion, on peut signaler l'incapacité pour un adolescent d'être en mesure de s'inscrire dans des échanges en groupe (troubles psychopathologiques avérés, retard mental...).

Chaque enregistrement de séance de groupe a fait l'objet d'une retranscription verbatim, relue par les animateurs des groupes considérés, afin de contribuer à compléter des fragments inaudibles ou identi- 
fier l'auteur de certaines séquences, et à apporter des éléments de contextualisation des propos au regard du déroulement du groupe et des événements qui le traversent entre deux séances ${ }^{6}$. La littérature ne comporte pas à notre connaissance de travaux reposant sur une telle méthodologie de recueil de données groupales, les travaux existants reposant généralement sur la dimension participative du chercheur?.

Ce sont les transcriptions qui constituent le matériel clinique objet de l'analyse: treize enregistrements de séances, avec des données exploitables pour neuf adolescents. L'analyse est basée sur la méthode d'analyse de discours de l'Interpretative Phenomenological Analysis (IPA) (Smith, Flowers et Larkin, 2009). Cette méthode s'inscrit dans une démarche inductive; conçue pour l'analyse de verbatim d'entretiens individuels, elle a été adaptée pour l'analyse de verbatim de groupe. Cette méthode qualitative cherche à saisir l'expérience subjective de l'individu à partir de deux caractéristiques:

- phénoménologique, elle vise une rencontre au plus près de l'expérience de l'individu. Cela sous-entend la nécessité de se départir des théories qui fondent notre compréhension des faits et de toute interprétation du discours et d'investir la posture du braqueting, ou mise en parenthèse, décrite par Husserl (1950) qui invite à partir à la rencontre du sujet, dans sa singularité et sa complexité;

- interprétative, cette méthode prend appui, dans sa conception originale, sur des entretiens semi-directifs conduits avec une personne à partir de questions liées à sa compréhension d'un aspect de son expérience.

Cette démarche confronte donc à une double herméneutique:

- le chercheur tente de donner du sens au sens que donne la personne sur la question; il y a sur ce point une divergence avec le recours à l'IPA dans la présente recherche, puisque les adolescents ne sont pas invités à donner du sens à une question particulière;

- l'écart de compréhension irréductible entre le chercheur et le sujet de la recherche sera utilisé pour porter un regard critique sur ce qui est dit et pour faire des liens qui n'existent pas explicitement dans le discours de l'autre.

6. Pour rappel, les deux groupes supports de la recherche ont un fonctionnement hebdomadaire, hors congés scolaires: l'enregistrement intervient donc toutes les dix séances en moyenne.

7. On peut se référer par exemple aux travaux de Vacheret $(2000,2014)$ ou Lo Piccolo (2015). 
Dans cette recherche, la méthode IPA a fait l'objet d'une adaptation de manière à saisir la singularité de l'expérience de l'adolescent à chaque temps de groupe enregistré, et l'évolution entre les séances. L'accent est mis sur le point de vue de l'adolescent: l'analyse est basée sur la production verbale de chaque adolescent en groupe et non sur la production des adolescents ou du groupe.

Le travail d'analyse s'est déployé selon deux grandes phases: à partir des données de l'étude pilote, basée sur trois adolescents ${ }^{8}$ et l'enregistrement de cinq séances de groupe, puis basée sur neuf adolescents pour l'ensemble des treize séances enregistrées. La première phase d'analyse conduit à la réalisation d'une grille à partir de laquelle, secondairement, l'ensemble des verbatim sera analysé. Au total, l'analyse repose donc sur 31 éléments de corpus, correspondant chacun au verbatim d'un adolescent pour une séance.

Pour la première phase, l'analyse du discours est réalisée en double aveugle par un binôme de chercheurs de l'équipe de recherche, pour chaque adolescent; le restant du groupe est envisagé comme le contexte dans lequel le discours est émis (background). Différentes étapes accompagnent cette première phase, qui mobilise la dimension longitudinale (comparaison entre les séances pour un même adolescent) et transversale (comparaison entre les adolescents). Elle conduit à l'établissement d'une grille d'analyse commune aux différents chercheurs impliqués, avec identification et définition de chaque thème et sous-thème. Les trois thèmes principaux retenus, au plus près de l'expérience des adolescents, sont les suivants: 1) la quête de repères; 2) la mise à l'épreuve de soi dans le lien aux autres; et 3) l'émergence et la construction des ressentis, émotions et affects (voir Tableau 2).

La seconde phase d'analyse prend appui sur la grille élaborée lors de la première phase, ainsi que sur la définition et les contours des thèmes et sous-thèmes qui la constituent. Elle porte sur l'ensemble des neuf adolescents de la recherche, et est réalisée en binôme de chercheurs, dans une démarche en double aveugle avec discussion des écarts et recherche d'un consensus interjuge, sous le regard des autres chercheurs de l'équipe de recherche.

8. Une étude pilote, portant sur trois adolescents, a permis de mettre à l'épreuve et d'ajuster le dispositif de la recherche et de consolider la structure méthodologique basée sur la démarche IPA. 
TABLEAU 2

Grille d'analyse thématique (version finale)

\begin{tabular}{|c|c|c|}
\hline Thème & Sous-thème & Définition \\
\hline \multirow[t]{4}{*}{ 1. La quête de repères } & $\begin{array}{l}1.1 \text { Les repères } \\
\text { tangibles }\end{array}$ & $\begin{array}{l}\text { Références ou demandes de réfé- } \\
\text { rences à des repères concrets } \\
\text { (dictionnaire, mesure, règles du } \\
\text { groupe...), ou explicitation d'une } \\
\text { absence de repères }\end{array}$ \\
\hline & $\begin{array}{l}\text { 1.2 Les repères } \\
\text { temporels }\end{array}$ & $\begin{array}{l}\text { Références à la temporalité en lien } \\
\text { avec le déroulement du groupe } \\
\text { (cadre horaire) ou avec le quotidien } \\
\text { ou l'histoire du sujet ou explicita- } \\
\text { tion d'une absence de repères }\end{array}$ \\
\hline & $\begin{array}{l}\text { 1.3 La confrontation } \\
\text { aux limites }\end{array}$ & $\begin{array}{l}\text { Énoncés faisant référence à son } \\
\text { propre positionnement par rap- } \\
\text { port aux limites et à la mise en } \\
\text { jeu des limites, avec les risques } \\
\text { de dépassement, sur le fond d'une } \\
\text { appropriation ou d'une tentative } \\
\text { d'appropriation des limites }\end{array}$ \\
\hline & $\begin{array}{l}\text { 1.4 La référence à } \\
\text { la loi (positive) }\end{array}$ & $\begin{array}{l}\text { Référence explicite à la loi et éven- } \\
\text { tuel positionnement: sur le versant } \\
\text { comportemental (respect vs trans- } \\
\text { gression), sur le versant cognitif } \\
\text { (connaissance ou non), ou sur le } \\
\text { plan du sens (référence symbolique } \\
\text { ou non) }\end{array}$ \\
\hline \multirow[t]{5}{*}{$\begin{array}{l}\text { 2. La mise à l'épreuve } \\
\text { de soi dans le lien } \\
\text { aux autres }\end{array}$} & $\begin{array}{l}2.1 \text { L'expression de } \\
\text { l'agressivité et } \\
\text { de la violence }\end{array}$ & $\begin{array}{l}\text { Propos exprimant de l'agressivité sur } \\
\text { le moment ou faisant référence à des } \\
\text { situations violentes (agies, subies ou } \\
\text { imaginées par l'adolescent) }\end{array}$ \\
\hline & $\begin{array}{l}2.2 \text { La prise de } \\
\text { position } \\
\text { subjective par } \\
\text { rapport aux actes }\end{array}$ & $\begin{array}{l}\text { Référence explicite aux actes } \\
\text { d'ordre sexuel commis par l'adoles- } \\
\text { cent; position réflexive }\end{array}$ \\
\hline & $\begin{array}{l}2.3 \text { La reconnais- } \\
\text { sance de l'autre } \\
\text { et le soin aux } \\
\text { autres }\end{array}$ & $\begin{array}{l}\text { Interaction entre les participants } \\
\text { ou référence à des interactions à } \\
\text { l'extérieur du groupe; implication } \\
\text { personnelle dans les relations }\end{array}$ \\
\hline & $\begin{array}{l}\text { 2.4 La capacité à } \\
\text { prendre soin } \\
\text { de soi }\end{array}$ & $\begin{array}{l}\text { Référence explicite de l'adolescent } \\
\text { à une préoccupation pour lui ou } \\
\text { pour les autres (expressions d'em- } \\
\text { pathie) }\end{array}$ \\
\hline & $\begin{array}{l}\text { 2.5 La quête de } \\
\text { reconnaissance } \\
\text { de soi }\end{array}$ & $\begin{array}{l}\text { Recherche de contact et d'attention } \\
\text { au travers de différentes modalités: } \\
\text { bruitages, agitation, commentaires, } \\
\text { tentative de s'accrocher au discours } \\
\text { des autres... (en deçà de la recon- } \\
\text { naissance de l'altérité de l'autre) }\end{array}$ \\
\hline
\end{tabular}


TABLEAU 2 (s u ite)

\begin{tabular}{|c|c|c|}
\hline Thème & Sous-thème & Définition \\
\hline \multirow[t]{6}{*}{$\begin{array}{l}\text { 3. L'émergence et la } \\
\text { construction des } \\
\text { ressentis, émotions } \\
\text { et affects }\end{array}$} & $\begin{array}{l}3.1 \text { Les vécus de } \\
\text { débordement }\end{array}$ & $\begin{array}{l}\text { Description des difficultés à conte- } \\
\text { nir le vécu interne et éventuels } \\
\text { aménagements et stratégies com- } \\
\text { portementales mises en œuvre pour } \\
\text { les limites }\end{array}$ \\
\hline & 3.2 Le corps qui parle & $\begin{array}{l}\text { Description des sensations phy- } \\
\text { siques explicitement liées ou non à } \\
\text { une émotion (à indiquer), ou des } \\
\text { manifestations somatiques }\end{array}$ \\
\hline & 3.3 Les échappées & $\begin{array}{l}\text { Toutes manifestations qui contri- } \\
\text { buent à se désengager de la rela- } \\
\text { tion à l'autre ou à soi-même: ratés } \\
\text { de langage, jeux de mots, humour, } \\
\text { oublis, évitements sélectifs, utili- } \\
\text { sation du «on»... }\end{array}$ \\
\hline & 3.4 La confusion & $\begin{array}{l}\text { Expression du sentiment de confu- } \\
\text { sion }\end{array}$ \\
\hline & $\begin{array}{l}3.5 \text { «J'ai pas le } \\
\text { choix»: le vécu } \\
\text { de contrainte et } \\
\text { d'impuissance }\end{array}$ & $\begin{array}{l}\text { Sentiment interne de ne pas avoir } \\
\text { prise sur un événement ou une } \\
\text { situation (peut reprendre des élé- } \\
\text { ments du thème } 2.1 \text { ); sentiment } \\
\text { d'impuissance }\end{array}$ \\
\hline & $\begin{array}{l}3.6 \text { L'expression des } \\
\text { éprouvés, } \\
\text { émotions et } \\
\text { affects }\end{array}$ & $\begin{array}{l}\text { Expression explicite des éprouvés, } \\
\text { émotions et affects ainsi que de } \\
\text { leur élaboration au travers de la } \\
\text { verbalisation }\end{array}$ \\
\hline
\end{tabular}

\section{Résultats}

De manière synthétique, sur un plan formel, les données cliniques recueillies apportent les éléments suivants:

- $100 \%$ des adolescents inclus dans la recherche sont des garçons;

- les adolescents de la recherche sont âgés, au moment de leur inclusion dans la recherche $(\mathrm{T})$, de 15 ans 2 mois à 19 ans 2 mois, et au second temps de recueil des données (RT), de 16 ans 2 mois à 19 ans 11 mois;

- ils ont tous fait l'objet d'une condamnation judiciaire pour des infractions sexuelles diverses (attouchements ou viols), commises sur des enfants prépubères, indifféremment filles ou garçons, et qui appartiennent au contexte familial de l'adolescent;

- ils n'ont pas d'antécédents judiciaires ni n'ont reçu de diagnostic psychiatrique; 
- l'histoire des adolescents rencontrés est marquée par des histoires de séparation, voire de deuil parental et par une instabilité des liens et des vécus traumatiques au sein de la famille;

- l'écart test-retest pour les épreuves projectives est en moyenne de huit mois;

- le nombre de séances de groupe considéré pour un même adolescent varie de trois à cinq séances (voir Tableau 3);

- les données complètes seront exploitées pour neuf adolescents (passation T-RT des épreuves projectives, participation à un minimum de trois séances enregistrées).

Dans cette section, les résultats issus de chaque catégorie de données cliniques (épreuves projectives, séances de groupe) seront présentés dans leurs grandes lignes. Puis la discussion s'ouvrira avec le croisement des données et la mise en évidence d'opérateurs de sens communs à partir de catégories transversales.

TABLEAU 3

Temps de groupe enregistré par adolescent $(T)$ et temporalité des séances (S)

\begin{tabular}{|c|c|c|c|c|c|}
\hline $\mathrm{T} \longrightarrow \mathrm{S}$ & S1 & S2 & S3 & S4 & S5 \\
\hline T1 & $\begin{array}{l}\text { Émile } \\
\text { Lilian } \\
\text { Serge }\end{array}$ & & & & \\
\hline T2 & Nicolas & $\begin{array}{l}\text { Émile } \\
\text { Lilian } \\
\text { Serge }\end{array}$ & & & \\
\hline T3 & $\begin{array}{l}\text { Charles } \\
\text { Corneille } \\
\text { David } \\
\text { Donald } \\
\text { Thibault }\end{array}$ & Nicolas & $\begin{array}{l}\text { Émile } \\
\text { Lilian } \\
\text { Serge }\end{array}$ & & \\
\hline T4 & & $\begin{array}{l}\text { Charles } \\
\text { Corneille } \\
\text { David } \\
\text { Donald } \\
\text { Thibault }\end{array}$ & Nicolas & $\begin{array}{l}\text { Lilian } \\
\text { Serge }\end{array}$ & \\
\hline T5 & & & $\begin{array}{l}\text { Charles } \\
\text { Corneille } \\
\text { David } \\
\text { Donald } \\
\text { Thibault }\end{array}$ & Nicolas & Serge \\
\hline $\mathrm{Nb}$ participants & 9 & 9 & 9 & 3 & 1 \\
\hline
\end{tabular}




\section{Données projectives}

Le traitement des données projectives met en évidence un certain nombre de mouvements singuliers entre les temps de T et RT témoignant de divers réaménagements au fil des mois. Ces mouvements peuvent être décrits à partir des cinq observatoires définis au préalable comme support à l'analyse et prennent appui sur les indicateurs préalablement établis.

1. Dynamique entre réalité interne et réalité externe. Deux tendances se dessinent dans la population des neuf adolescents: pour les uns, la production projective met en évidence une évolution favorable, avec en particulier une meilleure qualité des potentialités adaptatives; pour les autres, on assiste à une évolution plus limitée, avec une fragilisation dans l'investissement des repères.

On peut faire l'hypothèse que le travail thérapeutique engagé s'accompagne globalement d'une diminution de l'efficience défensive, en particulier liée à une meilleure appréhension des enjeux de la rencontre de l'autre (mobilisation de l'intersubjectivité). Les réaménagements nécessités par cette déstabilisation se traduisent dans une tension entre une perspective maturative et une perspective régressive: augmentation du nombre de réponses versus baisse du nombre de banalités et augmentation du nombre de refus au Rorschach, incidence plus marquée des procédés d'élaboration des récits renvoyant à la dynamique intersubjective (procédés série B) versus marques de désorganisation qui accompagnent ces procédés (procédés série E).

2. Problématique de séparation-différenciation. La fragilité narcissique des adolescents, dans ses traductions projectives, reste vive et le fonctionnement de leur vie psychique particulièrement réceptif aux sollicitations archaïques des planches de Rorschach (blanc, symétrie), qui exacerbent des modalités défensives rigides et autorisent peu de dégagement. En contrepoint, l'appui sur la symétrie autorise le déploiement de réponses de double, possiblement en mouvement, qui témoignent d'une relance de l'activité associative. Le support figuratif du TAT autorise davantage de reprise élaborative, avérée ou contrastée, pour une majorité des adolescents. Les évolutions les plus nettes entre test et retest, avec une gamme variée dans leurs traductions, s'expriment en lien avec la planche 13B, figurant un petit garçon auquel les adolescents s'identifient volontiers, sous la forme 
de récits qui intègrent davantage le recours à une figure secourable. Peut-on y voir l'effet du vécu de l'accueil dans le groupe et de la préoccupation des soignants et des autres adolescents?

3. Investissements oedipien versus narcissique. L'évolution entre test et retest se trouve particulièrement nette quant à l'investissement de la triangulation odipienne et de la tension avec les enjeux narcissiques qui la sous-tend (Pour David, à la planche 3B du TAT, la triangulation œdipienne est absente au $\mathrm{T}:$ «[...] à l'époque les paysans ils cultivaient leurs champs [...]»), alors qu'au RT, la tentative de construction se présente sur un mode très confus, une évocation du couple est possible, dans le contexte d'un lien de rivalité féminine (absence de référence à la différence des générations): "une dame qui est triste car le mari de la femme est enceinte, pis que elle elle a perdu son mari»). On peut percevoir en filigrane, au Rorschach et au TAT, de quelle manière la tension narcissique-objectal est centralement mise au travail, et observer la dynamique de changement qui en émerge. Les évolutions témoignent d'une forme de labilité des aménagements psychiques, sans que ne se dégage une ligne univoque. À nouveau, la qualité figurative du stimulus au TAT favorise le déploiement de la dynamique identificatoire: elle procure un appui permettant que s'opère un dégagement des enjeux de la construction narcissique-identitaire.

4. Liaison affect-représentation. Le RT marque sensiblement de nouveaux potentiels dans la capacité des adolescents à nommer, reconnaître et éprouver l'impact sensible de la réalité externe sur leur vie psychique (avec, dans l'exemple qui suit, la référence au toxique), ce constat devant être modulé selon les adolescents (Rorschach de Corneille, planche IX, émergente au RT de la possibilité de se laisser toucher dans une rencontre sensible avec le stimulus : (...) présence orange pour le feu, aussi du blanc, du rouge, du jaune. Mélange de couleurs chaudes. Si on veut du plastique ou de l'aluminium, plus une flamme verte, bleue, violet[te], enfin bref quelque chose qu'on voit que c'est toxique»). Au-delà, les adolescents se montrent davantage à même d'éprouver la consistance de l'affect à partir de la rencontre de la figure de l'autre dans les réponses au Rorschach ou les récits au TAT. La reconnaissance ou l'interrogation de l'affect, ou l'accès à l'ambivalence, constituent autant de ponts vers l'accès à une potentialité réflexive, et à un dégagement de la tyrannie qu'imposent des positions désaffectivées ou clivées. 
5. Mode de symbolisation de l'acte. Les données cliniques mettent en évidence une tension entre les deux épreuves projectives:

- au Rorschach, les potentiels de symbolisation restent contingents des fondements narcissiques, qui se présentent comme fragiles et limitent les évolutions, tangibles mais timides pour certains adolescents du fait de la fragilité de la construction de la représentation humaine, support de l'identité et des potentiels de subjectivation, et du faible investissement des mouvements affectés à ces représentations;

- au TAT en revanche, la mobilisation des potentiels de symbolisation entre $\mathrm{T}$ et RT est plus manifeste, en lien avec des sollicitations qui se rapportent directement au vécu des adolescents dans le registre de la violence ou de la sexualité et dont les récits portent trace de l'investissement (protocole de TAT de Serge, planche 8BM, au T: «Bah... c'est l'histoire [A2-1] d'un meurtre [E2-3], pis y a le monsieur qui... comment ça s'appelle? [CI-1] Qui regarde s'il y a de traces d' hémorragie [A2-2, E2-3] et le policier est devant [CL-2], il mène l'enquête, pis attend les résultats [CF-1]. Pis c'est tout. [CI-1] [E1-1]» et au RT: «C'est... C'est... [CI-3] c'est le médecin qui ouvre une personne, le ventre [E2-], comment il doit lui mettre un poumon, on peut mettre un poumon? [E4-2, CM-1] [...] Oui greffer un poumon au monsieur [CI-2] qui est couché et un jeune [CI-2] reste à côté et après le monsieur est de nouveau debout [A3-2] [E1-1]».) Par ailleurs, la rencontre avec le blanc, l'absence et le vide à la planche 16 autorise, au RT, l'ouverture sur des potentiels réflexifs en appui sur la mobilisation de figurations issues du monde interne de l'adolescent.

\section{Données groupales}

Le traitement des verbatims issus des groupes thérapeutiques pour neuf adolescents, réalisé à partir de la grille d'analyse thématique, permet de mettre en évidence un certain nombre de caractéristiques concernant l'évolution du discours des adolescents au fil des séances.

1. Repères et limites: du factuel au personnel, de l'objectif au sensible

L'évolution peut être énoncée dans une double valence qui témoigne de l'émergence d'un processus d'intériorisation. La mention explicite des repères, dans leur caractère factuel ou personnel tend à disparaitre 
(référence aux horaires, aux lieux...), au fil de la chronologie des groupes, au prix d'un vacillement et d'une insécurité le cas échéant, mais aussi au bénéfice d'une forme d'intériorisation (groupale?) qui permet de faire l'économie de leur verbalisation (possibilité de prendre appui sur des repères implicites, appartenant au cadre général du groupe). Concernant le rapport à la limite et à la loi, l'évolution d'une référence objective (liée à l'énoncé de la loi) à une référence subjective (appropriation de l'interdit) est notable, dans un mouvement qui donne à voir progressivement une forme d'incarnation personnelle et subjectivée de la loi.

2. Mise à l'épreuve dans le lien à l'autre: possible expression d'affect, fragilité du lien à l'autre et à soi

Les verbalisations tendent à évoluer vers une possible expression des affects chez les adolescents pour qui l'agir transgressif paraît constituer un équivalent de communication et d'adresse d'un vécu subjectif à l'égard de l'autre, qui s'exprime sur le mode de la souffrance et des angoisses de séparation/abandon. Les éprouvés liés aux actes transgressifs semblent progressivement prendre sens dans l'intersubjectivité par l'expression de la honte ou de la culpabilité. L'affrontement au groupe et à la groupalité, d'où émergent des interactions en termes d'identification ou de différenciation (sur le mode de la référence à la ressemblance ou à la dissemblance), signale des capacités à s'individuer et à s'autonomiser, prémisses d'un engagement dans le processus de subjectivation. En revanche, les aspects concernant la capacité à prendre soin de soi, et à se construire au travers d'une reconnaissance par l'autre (mobilisation de l'autre comme possible reflet de soi), apparaissent particulièrement en souffrance; ces aspects connaissent peu d'évolution, marquant une probable difficulté des adolescents à prendre soin d'euxmêmes, et une dépendance à l'autre massive, du fait de la prégnance du déficit narcissique.

3. Ressentis, émotions et affects: diminution des débordements, développement de stratégies de contenance des vécus traumatiques

Les vécus de débordement et les manifestations ou descriptions des sensations physiques qui accompagnent les émotions diminuent au fil de l'avancement du traitement. Les différentes échappées mobilisées témoignent de la prégnance de mécanismes de défense qui s'expriment 
tout au long du traitement et qui visent à tenir à distance les vécus traumatiques (ceux dont ils ont été victimes, ceux qu'ils ont infligés aux victimes) et la confusion qu'ils entraînent. Dans ce contexte, la capacité à nommer ses émotions et celles des autres demeure fragile. L'identification et la verbalisation des émotions et affects connaissent peu d'évolution, mais on relève un changement dans la capacité des adolescents à les contenir, à travers le développement de stratégies pour les canaliser. On note dans les manifestations langagières et infra-langagières un risque de débordement associé au passage à l'acte: celui-ci est explicitement craint par certains adolescents, décrit dans un sentiment de confusion concernant la sexualité, empêchant de penser et de sentir la limite, avec parfois l'expression de vécus somatiques insupportables. Avec la diminution du vécu de débordement et de la crainte du risque qu'il représente, les adolescents peuvent faire l'expérience dans les séances, avec l'étayage du groupe, de verbalisations d'une finesse parfois étonnante sur leurs vécus intimes. L'hyposensibilité aux affects apparaît comme un marqueur de la dysrégulation émotionnelle causée par le vécu traumatique présent chez tous les adolescents.

\section{Discussion}

La présente étude menée à partir des données cliniques recueillies auprès de neuf adolescents auteurs de violences sexuelles met en évidence l'importance de considérer le double registre dans lequel se déploie la dynamique psychique mobilisée à partir de l'agir transgressif. La dynamique psychique se déploie en effet tout à la fois du point de vue de l'intrapsychique (le monde interne de l'adolescent dans le rapport de soi à soi) et de l'intersubjectif (le monde de l'adolescent dans le rapport de soi à l'autre), sous-tendue par le registre du trans-subjectif (rapport aux enjeux de transmission entre les générations) dont quelques aspects émergent.

La mise en perspective des données cliniques longitudinales, issues des épreuves projectives et des verbatims de groupe, permet de faire émerger trois problématiques fédératrices pour la discussion: la construction des repères et la symbolisation de l'acte, la tension entre investissements narcissiques et objectaux, et la dynamique liaison déliaison dans les processus psychiques (destin de la pulsion, liaison de l'affect à la représentation). 
On a pu relever que les processus observés ne se présentent pas de manière homogène pour l'ensemble des adolescents rencontrés. Les perspectives tracées proposent une compréhension générale des processus mobilisés, sans que celle-ci n'épuise la spécificité du parcours de chacun des adolescents. Par ailleurs, les processus repérés ne se déploient pas de manière linéaire, et montrent leurs limites (sans doute à considérer également du point de vue de la temporalité réduite dans laquelle s'inscrit l'approche longitudinale), ce qu'illustrera tout particulièrement le développement de la troisième problématique.

\section{Construction des repères, symbolisation de l'acte et subjectivation}

Le temps de l'adolescence est un temps privilégié pour l'agir et pour la mise en jeu des «corps-en-acte» (Roman et Dumet, 2009), qui contribue au processus adolescent et à une élaboration du nouveau régime pulsionnel auquel le corps est soumis. L'agir violent sexuel, désigné socialement et symboliquement dans sa valence transgressive, est le plus souvent considéré comme témoin d'une perte de contrôle de l'adolescent sur son comportement, voire d'une échappée pulsionnelle incoercible; l'agir sexuel violent se trouve au centre de la préoccupation des professionnels responsable du soin aux adolescents... même s'il s'avère souvent difficile pour les professionnels de l'évoquer frontalement avec les adolescents (Roman, 2012). En filigrane, c'est la prise en compte de la souffrance de l'adolescent qui est visée, souffrance souvent masquée et peu accessible, ainsi que la prévention de la réitération de faits de violences sexuelles.

À cet égard, il est intéressant de suivre le cheminement psychique des adolescents au fil du traitement: comment peuvent-ils mettre à l'épreuve et/ou construire repères et limites? de quelle manière cette échappée de l'agir sexuel transgressif peut-elle faire l'objet d'une reprise symbolisante? En filigrane, c'est la question de l'évolution du processus de subjectivation qui se trouve interrogée. On assiste, au fil du processus de soin, à une appropriation et une internalisation des repères et, parallèlement, à une subjectivation des agirs transgressifs. Progressivement, l'explicitation des repères tend à s'estomper dans les échanges de groupe, tout comme évolue l'appui sur des repères formels aux épreuves projectives. Le dégagement d'un ancrage factuel ne semble pas sans risque, puisque des mouvements de vacillement, 
voire de désorganisation, accompagnent le passage de l'accrochage à des repères externes vers la subjectivation de ceux-ci. On peut comprendre que ce passage nécessite une armature psychique personnelle (narcissisme) suffisamment solide, afin de prendre le relais des repères externes. On observe par ailleurs qu'une grande fragilité du narcissisme s'exprime dans le groupe et relativement aux épreuves projectives, comme en témoignent les mouvements régressifs qui émaillent les expressions des adolescents.

Dans ce contexte, la place du groupe, et, plus généralement, de l'altérité, est significative. On peut en repérer la trace au travers de l'évolution de la capacité des adolescents à prendre place dans le groupe, comme sujet différencié et interlocuteur potentiel, et à s'inscrire dans l'ouverture à l'autre, à ses pensées et ses émotions: le groupe peut ainsi constituer un étayage précieux, confirmant des recherches antérieures (Grant et al., 2009; Lemitre et Coutanceau, 2006; Smaniotto et al., 2014). Par ailleurs, on peut établir un accès possible à une fonctionsupport du groupe au travers des échanges dans le groupe, dont témoigne l'évolution de la capacité des adolescents à se représenter une issue à la situation de solitude et/ou d'abandon d'un enfant. En effet, une meilleure capacité à se représenter un recours possible par rapport à un vécu dépressiogène émerge dans le temps du retest, devant la planche 13B du TAT qui mobilise la problématique de la solitude et/ ou de l'abandon. On peut faire l'hypothèse que ce mouvement se fonde sur l'expérimentation, au sein du groupe thérapeutique et en appui sur celui-ci, d'une solution de rechange aux vécus intimes liés à la séparation et à l'abandon. En contrepoint, il faut relever que le retest aux épreuves projectives donne l'occasion de l'investissement d'une voie de dégagement pour la quasi-totalité des adolescents face à la violence suggérée par le matériel (socialisation ou symbolisation de la violence), et pour certains d'entre eux face au rapproché sexuel homme-femme qui pourra faire l'objet d'une conflictualisation a minima. Enfin, l'évolution de la prise de position des adolescents devant la planche blanche du TAT témoigne de manière exemplaire d'une (re-)mise en jeu de la mobilisation des objets internes au travers de marques de subjectivation de l'expérience relativement au vide et à l'absence (symbolisation par la voie de l'autoreprésentation, du recours au narratif, de la mobilisation réflexive...). 


\section{Tension entre investissements narcissiques et objectaux}

L'ensemble des données recueillies dans le cadre de la recherche met en évidence la tension majeure entre les investissements narcissiques, qui rendent compte de l'identité (sentiment d'unité et de continuité de soi), et les investissements objectaux (capacité à se situer comme sujet dans la rencontre de l'autre). Cette tension se trouve par ailleurs au centre du processus adolescent (Jeammet, 1994), du fait des remaniements imposés par l'irruption de la puberté. Dans ce contexte, quelles voies de traitement de cette tension les adolescents engagés dans des violences sexuelles développent-ils au décours du processus thérapeutique? De quelle manière la dimension traumatique de ces violences adressées à l'autre victime vient-elle faire écho aux vécus traumatiques de l'enfance? Comment la rencontre de l'altérité, valorisée dans le processus groupal, autorise-t-elle une relance du processus adolescent?

La fragilité narcissique des adolescents parcourt les données de groupe et projectives, ce qui est congruent avec les données biographiques par ailleurs: cette fragilité, mise à l'épreuve des différents dispositifs mobilisés, emprunte deux voies distinctes au fil de l'observation longitudinale:

- elle fait l'objet de tentatives d'élaboration et de dégagement: pour une part, ces tentatives prennent forme au travers de la possibilité de s'affirmer comme sujet intègre et différencié (dans le groupe, dans les réponses ou récits aux épreuves projectives), et au travers de la possibilité d'expliciter subjectivement des vécus dans le registre de l'intrapsychique (souffrances et angoisses) et de l'intersubjectif (honte et culpabilité); pour une autre part, ces tentatives échouent, avec l'impossibilité d'investir une préoccupation pour soi-même, que l'on peut entendre comme l'écho ou la survivance de carences et/ou de vécus traumatiques précoces;

- elle sous-tend les tentatives d'affirmation des positions identificatoires, avec la mise en jeu du triple registre de différence (entre moi et l'autre, entre les sexes, entre les générations) fondateur de la conflictualité intersubjective; ainsi, les tentatives pour s'inscrire dans la relation à l'autre apparaissent déstabilisantes, voire menaçantes, et ouvrent possiblement sur une désorganisation des repères.

On comprend alors que l'invitation faite aux adolescents à s'inscrire dans un lien, par le dispositif thérapeutique de groupe, est prise dans un enjeu paradoxal, puisque le groupe apparaît comme un espace à 
double valence, soutenant et risqué. L'investissement du groupe se présente comme tributaire de l'aménagement des fondements narcissiques des adolescents, dont il apparaît nécessaire de prendre soin, au risque de renforcer et d'exacerber les défenses, ou de favoriser la confusion et la désorganisation (Chapelier, 2000).

\section{Liaison-déliaison dans les processus psychiques}

Comme cela a été rappelé, la vie psychique à l'adolescence est particulièrement soumise à des aléas au fil de son développement. En effet, la dynamique entre progression et régression y est centrale, l'investissement de mouvements régressifs permettant la réinstauration (réinvestissement) de zones de sécurité en appui sur des expériences connues et rassurantes, devant le risque de l'inconnu et de l'insécurité dans lesquels la puberté propulse l'adolescent. Ainsi, la dynamique entre la liaison (des émergences pulsionnelles à l'affect et à la représentation) et la déliaison (émergence brute des émergences pulsionnelles) infiltre le processus thérapeutique dans lequel est engagé l'adolescent. Dans ce contexte, quelle est la contribution de cette tension au sein du processus de symbolisation (liaison affect-représentation) et de subjectivation (devenir sujet de ses agirs) soutenu par le traitement psychothérapeutique? Comment le processus de liaison, au service des potentiels de changement de l'adolescent, peut-il être débordé par la force effractrice des vécus traumatiques infantiles, réactualisés par le traumatisme pubertaire (Gutton, 1991)?

Un observatoire privilégié de la dynamique liaison-déliaison concerne la place et le statut des affects et de leur inscription dans un système de représentation (processus de symbolisation). Les données recueillies, dans le groupe et aux épreuves projectives, mettent en évidence une évolution tangible dans l'expression et la gestion des affects. Progressivement, la nomination des affects (les siens, ceux des autres) s'avère possible, arrimée au vécu propre de l'histoire personnelle, dans la prise de position verbalisée relativement aux actes transgressifs commis, ou dans les productions de réponses ou de récits aux épreuves projectives. Le langage corporel, proche de l'éprouvé, ou l'évitement de toute marque d'affectation représentent les deux voies extrêmes de négociation de l'affect et cèdent la place à la possibilité (parfois fragile, parfois entravée) de nommer les émotions et les affects, de reconnaitre leur qualité et d'évaluer leur impact: un indicateur significatif à cet 
égard est la possibilité de l'adolescent d'inscrire l'ambivalence affective et la réflexivité dans les récits produits au TAT.

Cependant, les potentiels de symbolisation qui émergent chez les adolescents montrent leurs limites, d'une part dans la mesure des fragilités narcissiques relevées précédemment, et d'autre part dans la mesure de la charge effractrice des vécus traumatiques appelés par les sollicitations de la réalité externe (thématiques émergeant dans les groupes et les productions face aux épreuves projectives). Deux voies d'explicitation conjointes peuvent être envisagées à l'égard de ce constat:

- tout se passe comme si les émergences traumatiques actualisées dans le traitement (le risque de l'agir sexuel violent, de son échappée, de sa réitération...) venaient faire obstacle au processus de symbolisation, les potentiels de transformation sur la voie d'une mise en représentation se trouvant fragilisés; l'enjeu, pour l'adolescent, se limiterait alors à contenir l'émergence traumatique, à défaut de pouvoir en nommer l'impact subjectif;

- le processus associatif mobilisé dans le travail thérapeutique en groupe paraît contribuer à ouvrir la voie aux émergences traumatiques par l'affaiblissement de l'efficacité des mécanismes de défense; paradoxalement, la sollicitation de l'affect met en péril les capacités adaptatives des adolescents et ouvre une situation de danger pour l'équilibre de sa vie psychique.

C'est sans doute dans ce cadre que l'on peut comprendre la labilité des aménagements des adolescents auteurs de violences sexuelles pris en charge dans des groupes thérapeutiques, dans la mesure où ceux-ci sont pris dans une triple injonction, marquée par le processus de devenir sujet (processus de subjectivation) propre au travail psychique de l'adolescence: injonction développementale, avec la nécessité de transformation des investissements infantiles; injonction groupale ou injonction à l'interaction, qui fonde le processus thérapeutique; injonction sociale, avec la contribution à la sanction à laquelle renvoie l'inscription dans un dispositif de soin contraint.

\section{Psychodynamique des processus de changement}

Le développement des trois problématiques qui traversent les productions cliniques des adolescents permet la mise en évidence de quatre enjeux psychodynamiques majeurs qui sous-tendent les processus de changement. Ces enjeux peuvent soutenir une modélisation du proces- 
sus thérapeutique et contribuent à la définition d'indicateurs cliniques pour l'évaluation de ces processus dans le soin en groupe aux adolescents engagés dans des agirs violents sexuels:

- le travail de l'affect (éprouvé, reconnaissance, nomination, partage...), dont la place est centrale dans le travail thérapeutique (Chabert, 2017; Parat, 1995), avec l'importance de la prise en compte de l'affect comme levier thérapeutique et comme témoin de la place accordée à l'altérité au sein de la vie psychique;

- le travail du cadre, dont les déclinaisons au sein du groupe et au sein de la vie psychique des adolescents (limite, règles, interdit...) s'avèrent essentielles pour le développement de la capacité réflexive de l'adolescent; le travail du cadre assure le soutien des positions narcissiques et l'instauration d'une sécurité interne qui fait défaut dans l'expérience des adolescents;

- le travail du traumatisme, traumatisme dont l'impact parcourt l'histoire des adolescents, au double titre de traumatisme subi dans l'histoire infantile et pubertaire, et agi dans les violences sexuelles; l'accompagnement des vécus traumatiques permet de réinterroger les positions actives et passives dans le fonctionnement psychique des adolescents et dans leurs traductions comportementales, au service d'une capacité de symbolisation de l'acte;

- le travail du groupe, qui témoigne de la mise en jeu des fonctions d'étayage du groupe, ainsi que de celles d'accueil et de transformation des éprouvés, des affects et des représentations (Kaës, 1979), ainsi que de sa fonction de confrontation des narcissismes dans la mise en scène de l'altérité; la tension entre enjeux narcissiques et objectaux, au cœur du processus adolescent, trouve dans le travail du groupe un espace privilégié pour s'y déployer, au service de la subjectivation (Chapelier, 2000).

Ces quatre registres du travail psychique engagés dans le processus thérapeutique qualifient celui-ci au regard des transgressions pour lesquelles les adolescents ont été mis en cause et condamnés. En effet, la contribution traumatique des violences sexuelles à l'adolescence engage l'altérité, au travers d'une relation généralement familière, tout à la fois du point de vue de la victime de l'adolescent et de l'adolescent lui-même, dans l'histoire de ses propres liens. Le travail mené en groupe d'adolescents prend alors une valeur singulière, dans la mesure où le groupe se propose comme une autre scène, terrain d'expérimentation et de réparation possible de sa relation à l'autre; la charge émotionnelle 
dévastatrice du traumatisme rend délicat le travail sur les affects tant pour l'adolescent que pour les thérapeutes, puisque le risque lié à l'émergence de l'affect est celui du débordement versus de l'effondrement. Le travail sur le cadre se présente alors comme impératif, puisqu'il permet de valoriser les ressources des adolescents au travers de leur propre interrogation du cadre du groupe, et, au-delà, des repères qui structurent les liens.

À ce stade de la discussion apparaît un point aveugle, celui de la famille: la famille, considérée comme creuset du développement de la vie psychique et comme configuration systémique au sein de laquelle se jouent les investissements de l'adolescent, y compris les violents. L'hypothèse d'une fonction spécifique des violences sexuelles dans la dynamique familiale, à la fois du point de vue des enjeux de transmission générationnelle et de leur contribution à l'économie et la régulation des liens familiaux (de Becker, 2009; Lafortune, 2001; Roman et Ravit, 2006), mériterait d'être davantage explorée. Sans doute ce point aveugle constitue-t-il une trace du traumatisme, dans son ancrage intrafamilial, dont on peut faire le constat qu'il traverse nombre des histoires des adolescents auteurs de violences sexuelles. Doit-on alors penser la nécessité d'une expression de ces vécus traumatiques par l'adolescent, et d'une élaboration subjective suffisante de ceux-ci, comme un indicateur de la levée possible de la contrainte thérapeutique?

\section{Perspectives pour la pratique clinique}

L'étude sur l'évaluation des processus de changement des adolescents engagés dans des agirs violents sexuels pris en charge dans des groupes thérapeutiques met en évidence la complexité de l'identification des potentiels de changement des adolescents, qui reposent sur une suffisante plasticité de leur vie psychique dans ce temps charnière du développement. Outre l'intérêt de la mobilisation d'outils de repérage en appui sur les productions en groupe et les épreuves projectives, cette étude met en évidence des indicateurs sur lesquels repose l'évaluation $\mathrm{du}$ changement, autorisant, au-delà, une modélisation du processus thérapeutique en appui sur quatre voies principales: le travail de l'affect, le travail du cadre, le travail du traumatisme et le travail du groupe.

Si l'on considère l'agir violent sexuel à l'adolescence comme une occasion de relance du processus adolescent (Roman, 2012), il faut 
reconnaitre que la prise en charge groupale des adolescents constitue un outil pertinent et sensible pour l'accompagnement de celle-ci. L'accent a été mis sur la labilité des aménagements, sur l'absence de linéarité de l'évolution ainsi que sur la difficulté à déterminer des configurations spécifiques qui permettraient une catégorisation au sein de la population des adolescents: au regard des résultats de l'étude, les adolescents rencontrés émargent à des moments différents du processus, sur des voies successivement progrédientes et régrédientes, dans un arrimage et une quête d'accordage au groupe et aux figures qui le composent. Les processus qui sous-tendent le changement sont pluriels, et peuvent être compris comme le fruit d'aménagements singuliers qui résistent à une généralisation homogène d'un ou plusieurs profils d'adolescents.

Enfin, on peut relever de manière synthétique quelques perspectives et préconisations pour l'accompagnement psychothérapeutique groupal des adolescents auteurs de violences sexuelles avec quatre formulations pour fonder le traitement en groupe, peut-être spécialement au regard de la dimension contrainte du traitement sous mandat judiciaire, et de la réserve que suscite bien souvent l'accompagnement de ces adolescents.

\section{Soutenir et renforcer le narcissisme blessé des adolescents}

Au travers des données recueillies, la fragilité des assises narcissiques («le narcissisme blessé»; voir Ciavaldini, 2006; Roman, 2019) et la précarité de l'identité s'expriment de manière insistante. Le travail de groupe avec les adolescents permet de maintenir en tension deux exigences: d'une part celle d'assurer un environnement sécuritaire, à même d'accueillir l'adolescent dans sa souffrance et de favoriser un processus d'appartenance au groupe, et d'autre part celle de soutenir un accès à la différenciation, à l'individuation et à la subjectivation. L'enjeu du travail thérapeutique consiste alors à accompagner un renforcement du narcissisme de chaque adolescent en appui sur le travail groupal.

\section{Prendre soin du groupe et de la groupalité (adolescents, animateurs thérapeutes)}

Le processus de groupe implique un double emboîtement: l'adolescent dans le groupe, le groupe dans l'institution. Ce montage permet à l'adolescent de faire l'expérience d'une appartenance «génération- 
nelle», dans un contexte où l'on a pu mesurer la précarité de la structuration des repères, en particulier au travers des épreuves projectives (inscription des différents ordres fondateurs de la différence). Il est important que les adolescents soient invités à l'expérience d'un investissement soignant de la part des professionnels qui les accompagnent, en contrepoint des vécus carentiels qui ont été les leurs; il est également nécessaire que l'institution prenne soin du groupe et de ses membres (Simon, 1929), dans un investissement différencié entre professionnels et adolescents, afin de garantir une sécurité du cadre. La référence instituante de l'institution qui abrite le dispositif de soin représente une métaphore de la référence à la loi, pour les adolescents et pour les professionnels. Cette référence limitante et bienveillante fonde les potentiels d'empathie des professionnels à l'égard d'adolescents dont les agirs violents ne prêtent pas spontanément à la compassion.

\section{Respecter les mécanismes de défense dans la mobilisation $d u$ processus associatif}

L'accompagnement psychothérapeutique des adolescents auteurs de violences sexuelles implique un accueil suffisamment bienveillant des mouvements psychiques qui les habitent. La prégnance des expressions défensives peut être observée au travers des épreuves projectives et dans les manifestations en groupe; ces expressions signent la fragilité des organisateurs de la personnalité des adolescents et invitent les professionnels à une prudence dans le maniement des sollicitations portées par la méthode ou les outils qu'ils mettent en œuvre dans le groupe; cette prudence vaut également du point de vue de la mobilisation des interactions entre les adolescents, dont la dimension peu prévisible représente un risque (pour l'adolescent, pour le professionnel). Respecter les mécanismes de défense dans la mobilisation du processus associatif contribue à prendre soin de l'adolescent et à veiller à ce qu'il ne soit pas interpellé de manière trop frontale dans les zones les plus fragiles de son fonctionnement psychique en fonction de l'évolution du traitement. Une telle posture ne peut faire l'économie d'un temps suffisamment long du soin, qui autorise un dégagement de la contrainte à répéter la violence subie à partir de l'élaboration de celle-ci. 


\section{Accueillir les nécessaires mouvements régressifs comme des} leviers pour le changement

En contrepoint, l'accueil des mouvements régressifs, dont on a pu relever la prégnance au travers des différentes données recueillies, nécessite un arrimage suffisant des professionnels dans le dispositif de soin (analyse des enjeux inter-transférentiels entre les professionnels) et au sein de l'institution (travail de supervision): en filigrane, c'est la capacité d'accueil des mouvements régressifs des adolescents par les professionnels qui se trouve en jeu, ainsi que le soutien dans la transformation de ceux-ci. Ces mouvements régressifs doivent en effet être pensés, au-delà du caractère de décharge pulsionnelle dont ils témoignent, comme autant d'occasions de rencontrer l'adolescent dans ses investissements infantiles et souffrants, et de l'accompagner sur la voie de la subjectivation et du devenir-adulte.

Ces avancées de la recherche dans le champ de l'évaluation du processus thérapeutique au bénéfice d'adolescents engagés dans des agirs violents sexuels à partir d'un dispositif groupal mériteraient sans aucun doute d'être mises à l'épreuve dans le contexte d'autres dispositifs psychothérapeutiques. On peut assez aisément faire l'hypothèse que les indicateurs du processus thérapeutique identifiés trouveraient leur pertinence dans un dispositif de psychothérapie individuelle. Une telle mise en perspective permettrait par ailleurs d'affiner une compréhension du processus thérapeutique dans ce dispositif, et de spécifier la nature des enjeux propres au traitement individuel et groupal.

\section{Références}

Andronikof, A. et Réveillère, C. (2004). Rorschach et psychiatrie: à la découverte du malade derrière la maladie. Psychologie Française, 49(1), 95-110.

Auguste, D., Cardouat, T., Desguees, P., Laurent, C., Loger, P., Mallet, E.,... Zolezzi, C. (2010). La prise en charge psychiatrique pénitentiaire des auteurs d'agressions sexuelles: état des lieux, évolutions et enjeux (Module interprofessionnel de santé publique). Repéré à: https://documentation.ehesp.fr/ memoires/2010/mip/groupe_3.pdf

Balier, C., Ciavaldini, A. et Girard-Khayat, M. (1996). Rapport de recherche sur les agresseurs sexuels. Direction Générale de la Santé (France).

Brun, A. et Roussillon, R. (2016). Évaluation par le processus: la méthode à partir de dispositifs de psychothérapie institutionnelle. Dans A. Brun, R. Roussillon et P. Attigui (dir.), Évaluation clinique des psychothérapies psychanalytiques (p. 197-218). Paris, France: Elsevier-Masson 
Brun, A., Roussillon, R. et Attigui, P. (dir.) (2016). Évaluation clinique des psychothérapies psychanalytiques. Paris, France: Elsevier-Masson.

Chabert, C. (2017). Transferts d'affects. Dans J. André et C. Chabert (dir.), Vie et morts des affects (p. 15-37). Paris, France: PUF.

Chabert, C., Azoulay, C., Chretien, M., Guedeney, N., Jeamet, P., Aubin, J. P.,... Bréchon, G. (1990). Évaluation des processus de changement au cours du traitement d'adolescents psychotiques. La Psychiatrie de l'Enfant, 33(1), 189-285.

Chapelier, J.-B. (dir.) (2000). Le lien groupal à l'adolescence. Paris, France: Dunod.

Ciavaldini, A. (2006). La pédophilie, figure de la dépression primaire. Revue française de psychanalyse, 70(1), 177-195.

Collart, P., Depauw, Y. et Heremans, M. (2002). Aspects du traitement des abuseurs sexuels par une équipe hospitalière. Pensée plurielle, 4, 147-159.

de Becker, E. (2009). L'adolescent transgresseur sexuel. La Psychiatrie de l'Enfant, 52(2), 309-338.

Derezotes, D. (2000). Evaluation of yoga and meditation trainings with adolescent sex offenders. Child and Adolescent Social Work Journal, 17(2), 97-113.

Dwyer, R. G. et Boyd, M. S. (2009). Sex education for male adolescent sex offenders in a group setting led by general psychiatry residents: A literature review and example in practice. American Journal of Sexuality Education, 4(3-4), 208-224.

Ellerby, L., McGrath, R. J., Cumming, F. J., Burchard, B. B. et Zeoli, S. (2010). Pratiques courantes dans les programmes canadiens de traitement des agresseurs sexuels: sondage 2009 de la Safer Society Fondation. Repéré à: https://www. securitepublique.gc.ca/cnt/rsrcs/pblctns/2010-02-sss/2010-02-sss-fra.pdf

Emeraud, P. Y. (2001). Psychothérapie de groupe des auteurs d'agressions sexuelles: modalités, indications, objectifs, difficultés, limites... Dans Psychopathologie et traitements actuels des auteurs d'agression sexuelle (p. 257-262). Paris, France: John Libbey Eurotext et Fédération Française de Psychiatrie.

Emmanuelli, M. et Azoulay, C. (2008). Les épreuves projectives à l'adolescence. Paris, France: PUF.

Ertl, M. A. et McNamara, J. R. (1997). Treatment of juvenile sex offenders: A review of the literature. Child and Adolescent Social Work Journal, 14(3), 199-221.

Fischman, G. (2009). Fondements épistémologiques et modèle de validation. Dans G. Fischman (dir.), L'évaluation des psychothérapies et de la psychanalyse (p. 3-32). Paris, France: Elsevier-Masson.

Gamache, D., Diguer, L., Laverdière, O. et Rousseau, J.-P. (2014). Agressions sexuelles commises par des adolescents: relations entre des dimensions de l'organisation de la personnalité et des caractéristiques du délit. L'évolution psychiatrique, 79(4), 725-738.

Grant, J., Indermaur, D., Thornton, J., Stevens, G., Chamarette, C. et Halse, A. (2009). Intrafamilial adolescent sex offenders: Psychological factors and treatment issues. Australian Institute of Criminology.

Gutton, P. (1991). Le pubertaire. Paris, France: PUF.

Husserl, E. (1950). Idées directrices pour une phénoménologie et une philosopbie phénoménologique pures (traduit par P. Ricœur). Paris, France: Gallimard. 
Jeammet, P. (1994). Adolescence et processus de changement. Dans D. Widlöcher (dir.), Traité de psychopathologie (p. 687-726). Paris, France: PUF.

Kaës, R. (1979). Crise, rupture et dépassement. Paris, France: Dunod.

Kaës, R. (1993). Le groupe et le sujet du groupe: éléments pour une théorie psychanalytique du groupe. Paris, France: Dunod.

Laforest, S. et Paradis, R. (1990). Adolescents et délinquance sexuelle. Criminologie, 23(1), 95-116.

Lafortune, D. (2001). Abus sexuel juvénile et délinquance juvénile à la lumière des antécédents personnels et familiaux. Revue Internationale de Criminologie et de Police Technique, 54, 299-317.

Lemitre, S. et Coutanceau, R. (2006). Trouble des conduites sexuelles à l'adolescence. Clinique, théorie et dispositif psychothérapique. Neuropsychiatrie de l'enfance et de l'adolescence, 54, 183-188.

Levenson, J. S., Macgowan, M. J., Morin, J. W. et Cotter, L. P. (2009). Perceptions of sex offenders about treatment: Satisfaction and engagement in group therapy. Sexual Abuse: A Journal of Research and Treatment, 21(1), 35-56.

Lo Piccolo, G. (2015). Images violentes et violence de l'imaginaire: le Photolangage ( comme dispositif de transformation de la violence auprès d'adolescents agresseurs sexuels (Thèse de doctorat, Université Lumière-Lyon 2). Repéré à: http:// theses.univ-lyon2.fr/documents/lyon2/2015/lo_piccolo_g/pdfAmont/ lo_piccolo_g_these.pdf

McGarvey, J. et Lenaghan, M. (1996). A structured group approach with adolescent perpetrators. Child Abuse Review, 5, 203-213.

Parat, C. (1995). L'affect partagé. Paris, France: PUF.

Pelladeau, E. et Marchand, J. (2016). La perversion transitoire, un aménagement défensif? L'exemple de la violence sexuelle. Revue de l'enfance et de l'adolescence, 93(1), 187-200.

Pelladeau, E., Roques, M. et Pommier, F. (2015). Infractions sexuelles à l'adolescence et psychopathologie. Psychothérapies, 35(2), 117-129.

Perkins, D., Hammond, S., Coles, D. et Bishopp, D. (1998). Review of sex offender treatment programmes. Crowthorne, Royaume-Uni: High Security Psychiatric Services Commissioning Board.

Perron, R. (2010). La raison psychanalytique. Pour une science du devenir psychique. Paris, France: Dunod.

Piotrowski, Z.-A. (1936). On the Rorschach method and its application in organic disturbances of the central nervous system. Rorschach Research Exchange, 1, 23-40.

Roman, P. (2012). Les violences sexuelles à l'adolescence - comprendre, accueillir, prévenir. Paris, France: Elsevier-Masson.

Roman, P. (2015). Le Rorschach en clinique de l'enfant et de l'adolescent - Approche psychanalytique ( $2^{\mathrm{e}}$ éd.). Paris, France: Dunod.

Roman, P. (2016). Traumatisme et destin du sexuel infantile dans les agirs sexuels violents à l'adolescence. Psychologie clinique et projective, 22, 159-180.

Roman, P. (2019). Narcissisme blessé et identité en souffrance à l'adolescence. Filigranes, 28(1), 75-87. 
Roman, P. et Dumet, N. (2009). Des corps en actes: symbolisation / désymbolisation à l'adolescence. Cliniques Méditerranéennes, 79, 207-227.

Roman, P. et Melchiorre, M. (2015). Contribution des épreuves projectives à l'évaluation des processus de changement chez les adolescents auteurs de violence sexuelle - Une étude exploratoire. Psychologie clinique et projective, 21, 195-217.

Roman, P. et Ravit, M. (2006). La violence sexuelle dans la famille et la mise à l'épreuve des liens fraternels. Cabiers de Psychologie Clinique, 27, 11-27.

Roman, P. et Ravit, M. (2010). La subjectivation de l'agir sexuel violent à l'adolescence: les apports d'un questionnaire d'investigation clinique dans la rencontre de l'adolescent. Société et jeunesse en difficultés, 10. Repéré à http://sejed.revues.org/index6867.html

Simon, H. (1929). Aktivere Krankenbehandlung in der Irrenanstalt. Berlin et Leipzig, Allemagne: Walter de Gruyter.

Smaniotto, B., Réveillaud, M. et Félicier, M. (2014). Parcours thérapeutique d'un adolescent auteur de violences sexuelles pris en charge dans un dispositif de soins spécifiques. Neuropsychiatrie de l'enfance et de l'adolescence, 62, 379-385.

Smith, J., Flowers, P. et Larkin, M. (2009). Interpretative phenomenological analysis: Theory, method and research. Londres, Royaume-Uni: Sage.

Swart J., Bass, J. S. et Apsche, J. A. (2015). FMDT for sexual offending youth. Dans J. Swart, J. S. Bass et J. A. Apsche (dir.), Treating Adolescents with FamilyBased Mindfulness (p. 273-302). Springer.

Vacheret, C. (2000). Photo, groupe et soin psychique. Lyon, France: PUL.

Vacheret, C. (2014). Les groupes à médiation et la référence au modèle psychanalytique. Connexions, 102(2), 199-212.

Viens, S., Tourigny, M., Lagueux, F. et Loméga, E. (2011). Évaluation des effets d'une thérapie de groupe pour adolescents auteurs d'agressions sexuelles. Dans M. Tardif (dir.), L'agression sexuelle: Transformations et paradoxes (p. 307327). Montréal, Québec: Cifas-Institut Philippe-Pinel de Montréal.

Weiner, I. et Exner, J. (1991). Rorschach changes in long-term short-term psychotherapy. Journal of Personality Assessment, 56(3), 453-465.

\section{Processes of change for teenage sex offenders - Evaluation, clinical indicators, and perspectives on care in the context of group therapy}

ABSTRACT - This article presents the principal outcomes of research conducted in two clinics, one in Switzerland and one in France, that evaluated the processes of change in teenage sex offenders. The goal was to identify the main clinical indicators of these changes through a longitudinal study of a small cohort of adolescents $(\mathrm{N}=9)$ who were taking part in court-ordered group therapy. Data for this qualitative research were based on projective testing and retesting and on verbatim transcriptions of group sessions. Analysis of this data highlighted various clinical indicators that made it possible to 
sketch a model of the processes of change for adolescents. The information provided by this research helps outline prospects for psychotherapy with these young men.

KEYWORDS - Teenagers, sexual violence, psychotherapy, clinical indicators, evaluation.

\section{El proceso de cambio de los adolescentes autores de violencias sexuales. Evaluación, indicadores clínicos y perspectivas de cuidado en el marco de terapias de grupo}

RESUMEN - Este artículo presenta los principales avances derivados de una investigación conducida conjuntamente sobre dos sitios clínicos, en Suiza y en Francia, y consagrada a la evaluación de los procesos de cambio de los adolescente autores de violencias sexuales. El objetivo es identificar los indicadores clínicos principales de estos cambios, a partir de un estudio longitudinal sobre un pequeño número de adolescentes $(\mathrm{N}=9)$, comprometidos en un procedimiento psicoterapéutico bajo mandato judicial en grupo. El estudio se apoya en una metodología cualitativa que asocia, principalmente, dos tipos de datos clínicos: por un lado, pruebas proyectivas en test-retest $y$, por el otro, grabaciones textuales de los sesiones de grupo. A partir del análisis cruzado de estos datos, un cierto número de indicadores clínicos se ponen en evidencia, permitiendo esbozar una modelización de los procesos de cambio en los adolescentes. Más allá de esto, los elementos de comprensión que emergen de la investigación permiten trazar algunas perspectivas para el cuidado psicoterapéutico de los adolescentes.

PALABRAS CLAVE - Adolescente, violencias sexuales, psicoterapia, indicadores clínicos, evaluación. 